\title{
The Effect of Several Antibiotics on Lactococcus garvieae Isolated from Jordanian Dairy Products
}

\author{
Nizar Issa Alrabadi \\ Department Food Science and Nutrition, \\ Faculty of Agricultural Sciences, Jarash University, Jordan
}

Received 2012-09-20, Revised 2012-09-21; Accepted 2012-12-28

\begin{abstract}
This study investigated the antibiotic resistance profile of the $L$. garvieae isolated strains from Jordanian dairy products. Disk diffusion method was used. In particular, different antibiotics were tested against $L$. garvieae; the antibiotic disks were Trimethoprim $(5 \mu \mathrm{g})$, Clindamycin $(2 \mu \mathrm{g})$, Nitrofurantoin $(300 \mu \mathrm{g})$, Erythromycin $(15 \mu \mathrm{g})$, Ampicillin $(15 \mu \mathrm{g})$, Trimethoprim/Sulfamethoxazole $(1.25 / 23.75 \mu \mathrm{g}$ Polymyxin B $(300 \mathrm{U})$ and Tetracycline $(30 \mu \mathrm{g})$. The results indicated high and statistically significant effects of Trimethoprim, Nitrofurantoin and Trimethoprim/Sulfamthoxazole on L. garvieae. Trimethoprim had the greatest antimicrobial effect on $L$. garvieae strains. All of the strains were sensitive to this antibiotic. Trimethoprim showed $20 \mathrm{~mm}$ inhibition zone in some strains. On the other hand, there were no antibiotic effects of Clindamycin and Polymyxin B on L. garvieae. Slight and statistically insignificant effects were found of other tested antibiotics. The study recommended using Trimethoprim antibiotic against $L$. garvieae because it inhibited its growths in all isolated cultures.
\end{abstract}

Keywords: Jordanian Dairy Products, Antibiotics, Lactococcus Garvieae

\section{INTRODUCTION}

The genus Lactococcus is composed of seven species: L. garvieae, $L$. fujiensis L. piscium, $L$. chungangensis, L. plantarum, L. raffinolactis and $L$. lactis, (Cai et al., 2011). L. garvieae is the only kind that is classified as pathogenic (Facklam and Elliott, 1995; Vendrell et al., 2006). Thus, it is identified as an emerging zoonotic pathogen and the etiological agent of the lactococcosis (an emergent disease) in fish. Recently, L. garvieae has been the main cause of diseases affecting rainbow trout of fish in many countries around the world. Ghittino and Prearo (1992) argued that approximately $50 \%$ of total trout European production was lost due to diseases caused by this bacterium. Thus, the clinical and veterinary treatment of it is vital to study.

L. garvieae was first isolated from cases of bovine mastitis (Collins et al. 1983), thereafter, from clinical samples of human blood, urine and skin (Vinh et al.,
2006; Wang et al., 2007) and different kinds of food products such as fermented milk (El-Baradei et al., 2008) cheeses (Alegria et al., 2009; El-Baradei et al., 2007) and meat products (Aquilanti et al., 2007). Although, the pathogenic mechanisms of $L$. garvieae are poorly understood, this study investigated its sensitivity to different types of antibiotics. In fact, this study is important to determine whether the antibiotic resistance profile of the $L$. garvieae isolated from different Jordanian dairy products agree with the strains from different areas of the world. Antibiotic treatment of mastitis leads to significant increase in milk quantity and quality, lower somatic cell count and is likely associated with a reduction in prevalence of clinical mastitis among herds, which is economically beneficial (Oliver et al., 2003). Bacterial identification and susceptibility tests are important for selecting the appropriate antimicrobial agent affecting certain diseases (Gentilini et al., 2002). Examples of recent studies that conducted such tests are 
Murti and Kumar (2011) and Tirumalai and Prakash (2012). The rationale for this study is to determine the antibiotic which inhabits the growth of L. garvieae because it is becoming a risky bacterium that threatens the lives of humans and animals.

\section{MATERIALS AND METHODS}

\subsection{Isolated and Standard Strains}

Strains were isolated from Rayeb (fermented milk) which made from different types of milk including cow, sheep and goat milk. The standard strains were L. garvieae ATCC 43921 and ATCC 49156. They were obtained from the American Type Culture Collection (ATCC).

\subsection{Isolation, Purification and Identification of L. Garvieae}

Serial dilutions from samples $(11 \mathrm{~g})$ were emulsified in $99 \mathrm{~mL}$ of sterile $2 \%(\mathrm{w} / \mathrm{v})$ tri-sodium citrate solution and homogenized using an Ultra-Turrax mechanical blender. Serial dilutions were prepared in sterile $1 \%$ (w/v) peptone water, plated on M17 agar and incubated at $32^{\circ} \mathrm{C}$ for $48 \mathrm{~h}$. Then, they were purified by streaking four times on the same medium and temperature and tested by classical methods (gram staining, catalase test, growth at different concentrations of $\mathrm{NaCl}$ and different pH levels). Finally, the genomic DNA of the isolated strains was extracted using Sigma's GenElute Bacterial Genomic Kit, then it was identified through specific Polymerase Chain Reaction (PCR) test and specifically using the primers, (TTTGAGAGTTTGATCCTGG) and (AAGTAATTTTCCACTCTACTT) (Pu et al., 2002). Ten of them gave positive results.

\subsection{Antimicrobial Susceptibility Test}

The isolated and standard strains of $L$. garvieae were tested for their resistance to some antibiotics using disk diffusion method (Kirby et al., 1966); in this test, $2 \mathrm{~mL}$ of an overnight culture of $L$. garvieae were added to 20 $\mathrm{mL}$ of M17 agar medium and left to solidify. The antibiotic disks (Difco) were in contact with the agar, the plates were incubated at $32^{\circ} \mathrm{C}$ for $24 \mathrm{~h}$ and the antagonistic activity was evaluated by observing a clear zone of growth inhibition. The antibiotic disks were Trimethoprim $(5 \mu \mathrm{g})$, Clindamycin $(2 \mu \mathrm{g})$, Nitrofurantoin $(300 \mu \mathrm{g})$, Erythromycin $(15 \mu \mathrm{g})$, Ampicillin $(15 \mu \mathrm{g})$, Trimethoprim/Sulfamethoxazole $(1.25 \mu \mathrm{g} / 23.75 \mu \mathrm{g}$
Polymyxin B (300U) and Tetracycline $(30 \mu \mathrm{g})$. Duplicate of each antibiotic for each isolated strains were prepared and the average reading of each antibiotic was calculated. The inhibition zones for each antibiotic were determined in accordance with the Shryock and NCCLS (2002). Isolated strains were categorized as susceptible (sensitive) and resistant. T-test was used to determine the statistical significance of the inhibition zone. It was estimated through SPSS statistical package.

\section{RESULTS}

As shown in Fig. 1, ten isolated strains gave positive results in the PCR test and identified as L. garvieae. The antibiotic resistance profiles of $L$. garvieae isolated strains against eight antimicrobial agents are presented in Table 1 and Fig. 2. Our results indicated that Trimethoprim $(5 \mu \mathrm{g})$, Nitrofurantoin $(300 \mu \mathrm{g})$ and Trimethoprim/Sulfamethoxazole $(1.25 / 23.75 \mu \mathrm{g})$ had a great antibiotic action against all $L$. garvieae isolates. These results were statistically significant in all isolates and standard cultures. Furthermore, Trimethoprim $(5 \mu \mathrm{g})$ had the greatest antimicrobial effect on $L$. garvieae isolates. Thus, it showed $20 \mathrm{~mm}$ inhibition zone in some isolates. On the other hand, Clindamycin $(2 \mu \mathrm{g})$ and Polymyxin B (300U) had no effect on different $L$. garvieae isolates. $50 \%$ of $L$. garvieae isolates exhibited sensitivity to Erythromycin $(15 \mu \mathrm{g}) ; 58 \%$ of the isolates exhibited sensitivity to Ampicillin $(15 \mu \mathrm{g})$ while only $42 \%$ of them exhibited sensitivity to Tetracycline (30 $\mu \mathrm{g})$. However, all the reported values of these three antibiotics were statistically insignificant.

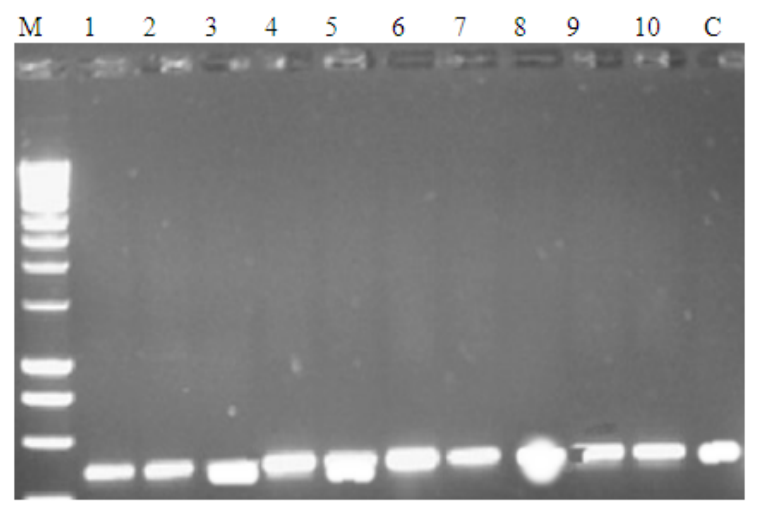

Fig. 1. Identification of L. garvieae by specific PCR assay 482pb M: Smartladder, Line: 1-10 isolated cultures, C: positive control 


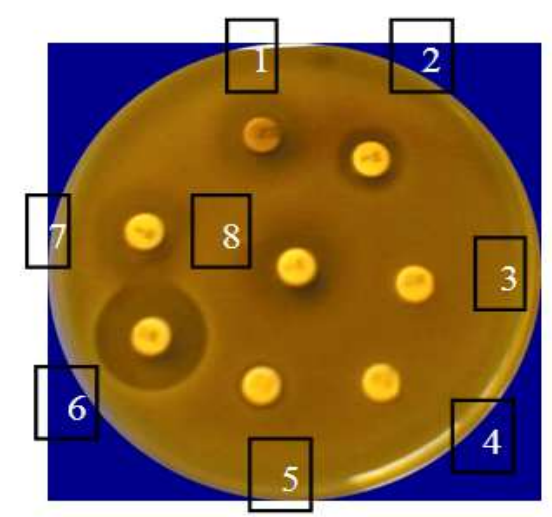

(a)

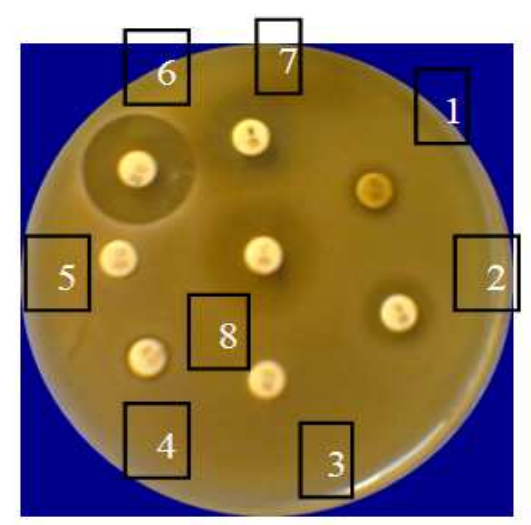

(b)

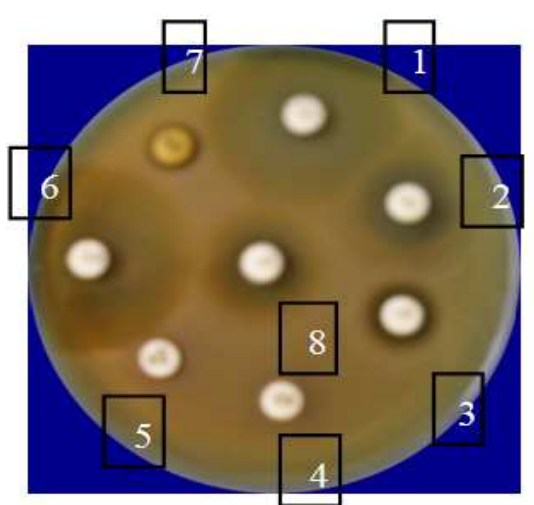

(c)

Fig. 2. Effect of different antibiotic on L.garvieae a, b, c denote randomly selected medias inculcated by L. garvieae and treated by different antibiotics. 1: Trimethoprim/Sulfamethoxazole, 2: Nitrofurantoin, 3: Erythromycin, 4: Polymyxin, 5: Clindamycin, 6: Trimethoprim, 7: Ampicillin, 8: Tetracycline

Table 1. Effect of different antibiotic on L.garvieae

\begin{tabular}{|c|c|c|c|c|c|c|c|c|c|}
\hline \multirow[b]{2}{*}{$\begin{array}{l}\text { Strain } \\
\text { number }\end{array}$} & \multicolumn{9}{|c|}{ Antibiotic and its concentration } \\
\hline & $\begin{array}{l}\text { Strain } \\
\text { source }\end{array}$ & $\mathrm{T}(5 \mu \mathrm{g})$ & $\mathrm{CD}(2 \mu \mathrm{g})$ & NF $(300 \mu \mathrm{g})$ & $\mathrm{E}(15 \mu \mathrm{g})$ & AM $(10 \mu \mathrm{g})$ & $\begin{array}{l}\mathrm{T} / \mathrm{S}(1.25 \mu \mathrm{g} / \\
23.75 \mu \mathrm{g})\end{array}$ & PB (300U) & $\mathrm{TE}(30 \mu \mathrm{g})$ \\
\hline \multicolumn{10}{|c|}{ Inhabitation Zone (mm) } \\
\hline 1 & Cow & $+16^{* *}$ & $-0^{\mathrm{NS}}$ & $+12^{*}$ & $+9^{\mathrm{NS}}$ & $+9^{\mathrm{NS}}$ & $+14^{* *}$ & $-0^{\mathrm{NS}}$ & $+8^{\mathrm{NS}}$ \\
\hline 2 & Cow & $+14^{* *}$ & $-0^{\mathrm{NS}}$ & $+11^{*}$ & $+8^{\mathrm{NS}}$ & $+9^{\mathrm{NS}}$ & $+13^{*}$ & $-0^{\mathrm{NS}}$ & $-0^{\mathrm{NS}}$ \\
\hline 3 & Cow & $+15^{* *}$ & $-0^{\mathrm{NS}}$ & $+10^{*}$ & $+6^{\mathrm{NS}}$ & $+9^{\mathrm{NS}}$ & $+11^{*}$ & $-0^{\mathrm{NS}}$ & $-0^{\mathrm{NS}}$ \\
\hline 4 & Sheep & $+18^{* *}$ & $-0^{\mathrm{NS}}$ & $+14^{* *}$ & $-0^{\mathrm{NS}}$ & $+8^{\mathrm{NS}}$ & $+13^{*}$ & $-0^{\mathrm{NS}}$ & $-0^{\mathrm{NS}}$ \\
\hline 5 & Sheep & $+13^{* *}$ & $-0^{\mathrm{NS}}$ & $+10^{*}$ & $-0^{\mathrm{NS}}$ & $+7^{\mathrm{NS}}$ & $+12^{*}$ & $-0^{\mathrm{NS}}$ & $+7^{\mathrm{NS}}$ \\
\hline 6 & Sheep & $+17^{* *}$ & $-0^{\mathrm{NS}}$ & $+11^{*}$ & $+7^{\mathrm{NS}}$ & $-0^{\mathrm{NS}}$ & $+12^{*}$ & $-0^{\mathrm{NS}}$ & $+8^{\mathrm{NS}}$ \\
\hline 7 & Sheep & $+18^{* *}$ & $-0^{\mathrm{NS}}$ & $+10^{*}$ & $+7^{\mathrm{NS}}$ & $-0^{\mathrm{NS}}$ & $+14^{* *}$ & $-0^{\mathrm{NS}}$ & $+8^{\mathrm{NS}}$ \\
\hline 8 & Goat & $+19^{* *}$ & $-0^{\mathrm{NS}}$ & $+11^{*}$ & $-0^{\mathrm{NS}}$ & $+7^{\mathrm{NS}}$ & $+12^{*}$ & $-0^{\mathrm{NS}}$ & $-0^{\mathrm{NS}}$ \\
\hline 9 & Goat & $+16^{* *}$ & $-0^{\mathrm{NS}}$ & $+13^{*}$ & $+7^{\mathrm{NS}}$ & $+7^{\mathrm{NS}}$ & $+14^{* *}$ & $-0^{\mathrm{NS}}$ & $-0^{\mathrm{NS}}$ \\
\hline 10 & Goat & $+20^{* *}$ & $-0^{\mathrm{NS}}$ & $+11^{*}$ & $-0^{\mathrm{NS}}$ & $-0^{\mathrm{NS}}$ & $+13^{*}$ & $-0^{\mathrm{NS}}$ & $+7^{\mathrm{NS}}$ \\
\hline 11 & ATCC 43921 & $+15^{* *}$ & $-0^{\mathrm{NS}}$ & $+12^{*}$ & $-0^{\mathrm{NS}}$ & $-0^{\mathrm{NS}}$ & $+14^{* *}$ & $-0^{\mathrm{NS}}$ & $-0^{\mathrm{NS}}$ \\
\hline 12 & ATCC 49156 & $+14^{* *}$ & $-0^{\mathrm{NS}}$ & $+11^{*}$ & $-0^{\mathrm{NS}}$ & $-0^{\mathrm{NS}}$ & $+13^{*}$ & $-0^{\mathrm{NS}}$ & $-0^{\mathrm{NS}}$ \\
\hline \multirow{2}{*}{\multicolumn{2}{|c|}{$\begin{array}{l}\% \text { of sensitive } \\
\text { L.garvieae strains }\end{array}$}} & $100 \%$ & $0 \%$ & $100 \%$ & $50 \%$ & $58 \%$ & $100 \%$ & $0 \%$ & $42 \%$ \\
\hline & & & & & & & & & \\
\hline
\end{tabular}




\section{DISCUSSION}

The results indicated that Trimethoprim was having best antimicrobial activity against $L$. garvieae because it inhibited its growths in all isolated strains. On the other hand, the study recommended not using Clindamycin, Polymyxin B, Erythromycin, Ampicillin and Tetracycline because they showed slight or no effect at all against $L$. garvieae. Comparing to other studies which used similar concentrations of antibiotics, our results were consistent with (Elliot and Facklam., 1996; Zlotkin et al., 1998), in that all the L. garvieae strains were resistant to clindamycin antibiotic and compatible with Raissy and Ansari (2011) that not all L. garvieae strains were sensitive to Ampicillin. Additionally, our results agreed with Baeck et al., 2006 that the isolated strains of $L$. garvieae were sensitive to Nitrofurantoin. However, we contradicted Tanrikul and Gultepe (2011) who found that L.garvieae is sensitive to Ampicillin. Understanding the resistance profile of $L$. garvieae is crucial given its severe pathogenic effects. Hence, this study explored the most effective drug against it.

\section{CONCLUSION}

Bacterial susceptibility tests are vital for selecting the most effective antibiotic eliminating certain diseases. This study investigated the effect of several antibiotics on L. garvieae isolated from Jordanian dairy products. Our findings indicated that Trimethoprim, Nitrofurantoin and Trimethoprim/Sulfamethoxazole had high and statistically significant antimicrobial effect on $L$. garvieae, however, Trimethoprim showed the greatest effect.

\section{REFERENCES}

Alegria, A., P.A. lvarez-Martin, N. Sacristan, E. Ferna'ndez and S. Delgado et al., 2009. Diversity and evolution of the microbial populations during manufacture and ripening of Casin, a traditional Spanish, starter-free cheese made from cow's milk. Int. J. Food Microbiol., 136: 44-51.PMID: 19822375

Aquilanti, L., C. Garofalo, A. Osimani, G. Silvestri and C. Vignaroli et al., 2007. Isolation and molecular characterization of antibiotic-resistant lactic acid bacteria from poultry and swine meat products. J. Food Protection, 70: 557-565. PMID: 17388042

Baeck, W.G., H.J. Kim, K.D. Gomez and C.S. Park, 2006. Isolation and characterization of Streptococcus sp. from diseased flounder (Paralichthys olivaceus) in Jeju Island. J. Vet Sci., 7: 53-58. PMID: 16434850
Cai, Y., J. Yang, H. Pang and M. Kitahara, 2011. Lactococcus fujiensis sp. Nov, a lactic acid bacterium isolated from vegetable matter. Int. J. Syst. Evolu. Microbiol., 61: 1590-1594.PMID: 20675438

Collins, M.D., J.A.E. Farrow, B.A. Phillips and O. Kandler, 1983. Streptococcus garvieae sp. nov. and Streptococcus plantarum sp. nov. J. Gen. Microbiol., 129: 3427-3431. PMID: 6663283

El-Baradei, G., A. Delacroix-Buchet and J. C. Ogier, 2007. Biodiversity of bacterial ecosystems in traditional Egyptian Domiati cheese. Appl. Environ. Microbiol., 73: 1248-1255.

El-Baradei, G., A. Delacroix-Buchet and J.C. Ogier, 2008. Bacterial biodiversity of traditional Zabady fermented milk. Int. J. Food Microbiol., 121: 295301. DOI: 10.1016/j.ijfoodmicro.2007.11.014

Elliot, J.A. and R.R. Facklam, 1996. Antimicrobial susceptibilities of Lactococcus lactis and Lactococcus garvieae and a proposed method to discriminate between them. J. Clin. Microbiol., 34: 1296-1298. PMID: 8727924

Facklam, R. and J.A. Elliott. 1995. Identification, classification and clinical relevance of catalasenegative, Gram-positive cocci, excluding the streptococci and enterococci. Clin. Microbiol. Rev., 8: 479-95.PMID: 8665466

Gentilini, E., G. Denamiel A. Betancor M. Rebuelto and M. Rodriguez et al., 2002. Antimicrobial susceptibility of coagulase-negative staphylococci isolated from bovine mastitis in Argentina. J. Dairy Sci., 85: 1913-1917.PMID: 12214983

Ghittino, C. and M. Prearo, 1992. Report of Streptococcosis in rainbow trout (Oncorhynchus mykiss) in Italy: preliminary note. Boll. Soc. Patol. Ittica., 8: 4-9.

Kirby, W.M.M., A.W. Bauer, J.C. Sherris and M. Truck, 1966. Antibiotic susceptibility testing by a standardized single disk method. Am. J. Clin. Pathol, 45: 493-496. PMID: 5325707

Murti, K. and U. Kumar, 2011. Antimicrobial activity of Ficus benghalensis and Ficus racemosa roots L. Am. J. Microbiol., 2: 21-24. DOI: 10.3844/ajmsp.2011.21.24

Oliver, S.P., M.J. Lewis, B.E. Gillespie H.H. Dowlen and E.C. Jaenicke et al., 2003. Prepartum antibiotic treatment of heifers: Milk production, milk quality and economic benefit. J. Dairy Sci., 86: 11871193.PMID: 12741543 
Pu, Z.Y., M. Dobos, G.K. Limsowtin and I.B. Powell, 2002. Integrated polymerase chain reaction-based procedures for the detection and identification of species and subspecies of the gram-positive bacterial genus Lactococcus. J. Appl. Microbiol., 93: 353361. PMID: 12147085

Raissy, M. and M. Ansari, 2011. Antibiotic susceptibility of Lactococcus garvieae isolated from rainbow trout (Oncorhynchus mykiss) in Iran fish farms. African J. Biotechnol., 10: 1473-1476.

Shryock, T.R. and NCCLS, 2002. Performance standards for antimicrobial disk and dilution susceptibility tests for bacteria isolated from animals: approved standard. 2nd Edn., NCCLS, ISBN-10: 1562384619, pp: 86.

Tanrikul, T. and N. Gultepe, 2011. Mix Infection in Rainboe Trout (Oncorhynuchus mykiss Walbaum): Lactococcus garvieae and Vibrio anguillarum O1. J. Animal Vet. Advance, 10: 1019-1023.

Tirumalai, P.S. and S. Prakash, 2012. Antibiotic resistance in co-culture biofilm of Listeria monocytogenes J0161. Am. J. Microbiol., 3: 7-17. DOI: 10.3844/ajmsp.2012.7.17
Vendrell, D., J.L. Balcazar, I. Ruiz-Zarzuela, I. de Blas and O. Girones et al., 2006. Lactococcus garvieae in fish: A review. Comparative Immunol. Microbiol. Infectious Dis., 29: 177-198. DOI: 10.1016/j.cimid.2006.06.003

Vinh, D.C., K.A. Nichol, F. Rand and J.M. Embil, 2006. Native valve bacterial endocarditic caused by Lactococcus garvieae. Diagnostic Microbiol. Infectious Dis., 56: 91-94. DOI: 10.1016/j.diagmicrobio.2006.02.010

Wang, C.Y.C., H.S. Shie and S.C. Chen, 2007. Lactococcus garvieae infections in humans: Possible association with aquaculture outbreaks. Int. J. Clinical Practice, 61: 68-73. PMID: 16704679

Zlotkin, A., A. Eldar, C. Ghittino and H. Bercovier. 1998. Identification of Lactococcus garvieae by PCR. J. Clinical Microbiol., 36: 983-985. 